\title{
THE MANAGEMENT PROCESS OF ORGANIZATIONS ADOPTING MULTI-AGENT SYSTEMS THAT SUSTAIN THE ACCELERATION OF DISTRIBUTED NEURAL NETWORKS AT SCALE
}

\author{
Aurel Mihail ȚîȚU ${ }^{* a, b}$, Alexandru STANCIU ${ }^{c}$ \\ ${ }^{a}$ Lucian Blaga University of Sibiu, Romania \\ ${ }^{b}$ Academy of Romanian Scientists, Bucharest, Romania \\ ${ }^{c}$ Microsoft Romania, Bucharest
}

DOI: $10.24818 / \mathrm{IMC} / 2020 / 01.11$

\begin{abstract}
The paper intends to investigate the management process for adopting multi-agent systems and their impact on a data-driven organization. While enabling distributed artificial intelligence to process data, today's organizations gain additional knowledge over insights provided by artificial neural networks present through multi-agent systems. Distributed neural networks revolutionize the decision-making, prediction ability, and real-time reactivity systems of the mobility and industrial landscape of present times. Contributions and conclusions emerge from leveraging impact and observations from various use cases, and critical aspects regarding the management process are revealed and highlighted. The purpose is to uncover technological, legal, ethical, and social aspects and stimulate the adoption of distributed artificial intelligence through the joint development of machine learning through multi-agent systems.
\end{abstract}

KEYWORDS: distributed artificial intelligence, multi-agent systems, machine learning, artificial neural networks

\section{INTRODUCTION}

Line of business decision-makers with a strong focus on outcomes are increasingly accelerating the adoption of artificial intelligence functions such as voice recognition, chatbots, digital assistants, and business intelligence software that increases their operational efficiency and capabilities. Knowledge-based organizations rely on processes that reveal the power of data lake and Big Data architecture, high-performance computing, and distributed computing through multi-agent systems. Human designed strategy and wisdom emerge through mathematical, statistical, and software development by using and processing large amounts of data ranging up to petabytes/day. In the pursuit of improving computational models emerging through software 2.0 systems, artificial neural networks become more productive. Why do multi-agent systems play an essential role in the development of artificial neural networks? Artificial intelligence platforms used for processing petabytes in scale per day are dependent on the logical, time-to-insights dependent processes for providing real-time results. Many methodologies that approach big-data concepts such as data lakes include multi-agent systems that divide complex processes into smaller, separated parts. Each agent acts simultaneously as an autonomous entity is independent of its superiors and as a collaborative part of a system of agents. Dynamic structures with the ability to adapt to the needs of processing specific tasks and multi-agent systems are essential aspects of distributed artificial intelligence systems. The different scenarios that arise in the agent to agent, agent to agents, and agents to agents interaction reveal the need for a rapidly changing and adapting a model that encapsulates the

*Corresponding author. E-mail address: mihail.titu@ulbsibiu.ro 
negotiation, communication, and political interaction of agents. Each has its owner bias and influence. From a legacy perspective, agents that support decision-making, data management, or analytics are solely dependent on human creativity and control. The way they create, send, and interpret messages or signals from the environment or other agents, compute and act are done in many ways. The challenge that the present study paper addresses are a future vision of distributed, multi-layer, artificial neural networks that include multi-agent systems.

\section{CONCEPTS, CLASSIFICATION AND CONTEXT FOR MULTI-AGENT SYSTEMS}

Agents are autonomous entities capable of decision-making on an individual level and emerge due to the increasing demand in distributed artificial intelligence, compute and decision-making capability of informational systems. A human-centric approach of the digital era demands the development of distributed artificial intelligence that evolves, communicates, and empowers humans to achieve and extract knowledge from their data. A multi-agent system in the context of artificial intelligence envisions the power of using artificial intelligence to optimize proactive, reactive, and collaborative decision-making for actions, recommendations, and predictions. In most of the use cases, asynchronous operations perform due to the different environment inputs and the pre-defined problem-solving methodology. Multi-agent systems, in the context of machine learning and model recognition or adjustment, are based on an existing and architected flow down. The flexibility of multi-agent systems and the ability to adapt to their environment is highly dependent on the use case and the expected output. Everyday use cases involve classification, pattern recognition, control, prediction, optimization, or data mining. Machine learning capabilities paired with multi-agent systems broaden the horizon from a single action type to multi-dimensional classification capability, enhancing the computational capability to unprecedented levels.

\subsection{Artificial neurons, the basis of neural networks}

At its essence, an artificial neuron is the primary component of a neural network and belongs to one of the three layers forming a neural network:

- Input layer

- Hidden layer

- Output layer

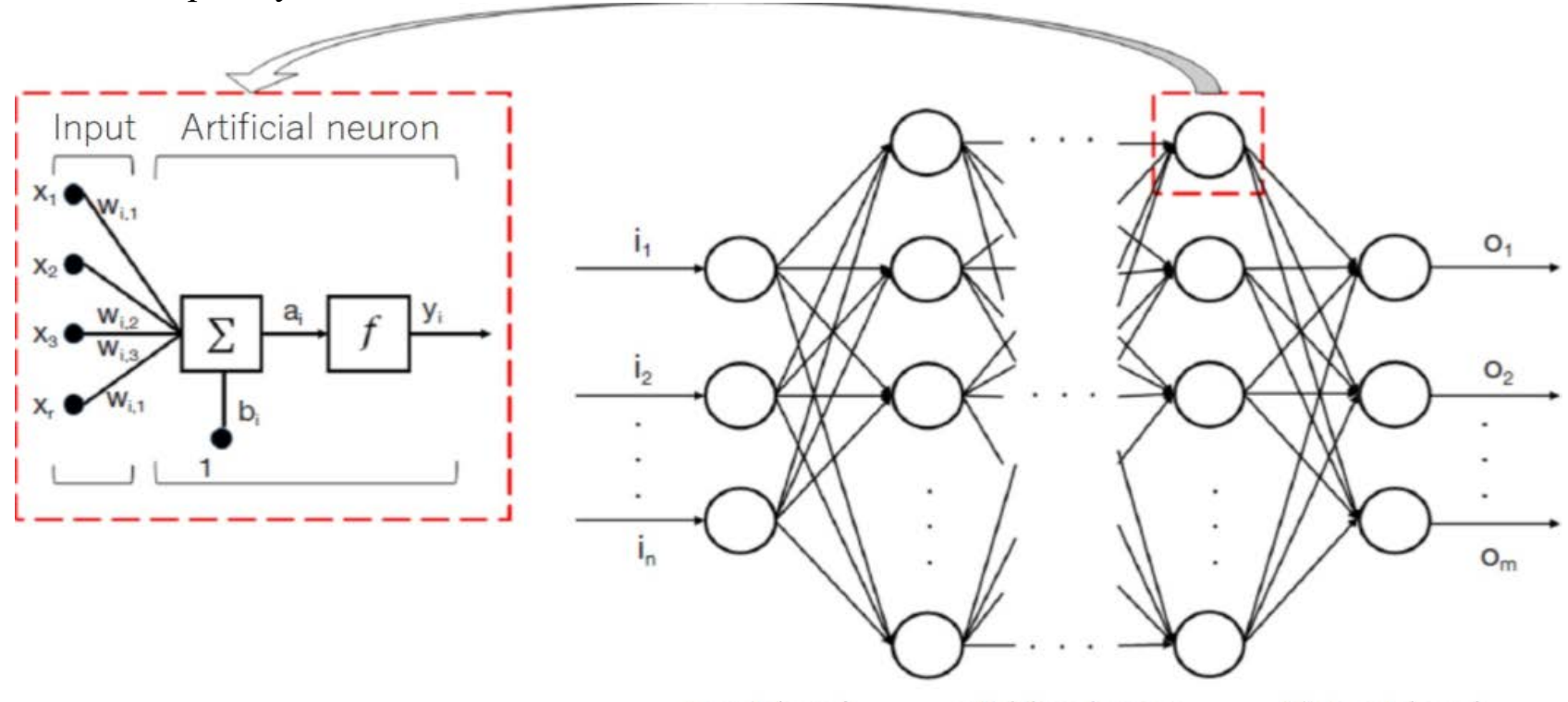

Input level Hidden layers Output level

Figure 1. Layers of Artificial Neural Networks

Source: adapted from Oustimov \& Vincent (2014) 
From input towards output, the multi-layer neural network, also known as a feed-forward neural network, has a single direction (Nurfikri, 2020). Deep neural networks have multiple hidden layers and can communicate outputs based on a multi-layer processing and feed-forward back propagation methodology. Such a network that uses both directions is called a feed-back neural network and has a higher level of complexity, slowing down the computational speed at the cost of increasing the accuracy of results.

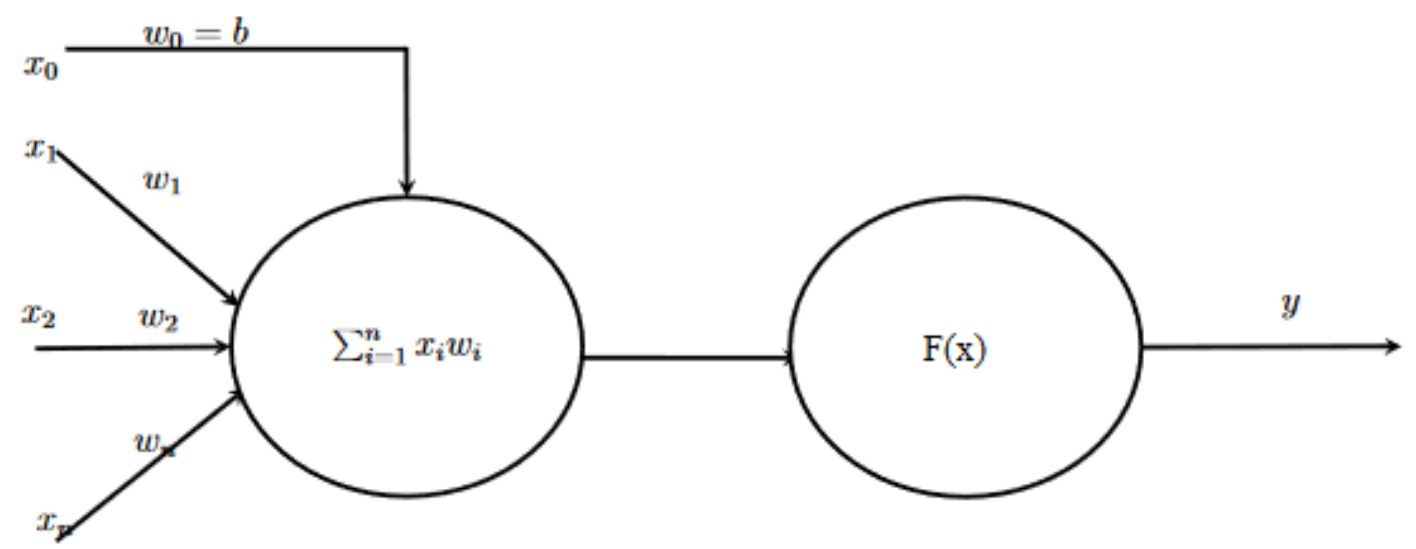

Figure 2. Artificial Neuron Logical and Mathematical View

Source: adapted from Learning Activation Functions In Deep Neural Networks (2017)

Within the neuron architecture, inputs show as $\mathrm{x} 1, \mathrm{x} 2, \ldots, \mathrm{xn}$ whereby each input is multiplied with w1, w2, ... wn representing weights of each input (Farhadi, 2017). Bias, displayed as a numeric value, for each neuron, ought to be added to the sum of inputs, in the following form(Shiruru, 2015):

$$
x=\sum_{i=1}^{n} x_{i} w_{i}+b
$$

$\mathrm{F}(\mathrm{x})$ is called activation function and can be expressed in multiple ways, depending on the use case:
a) Linear: $\mathrm{F}(\mathrm{x})=\mathrm{x}$;
b) Uni-Polar Sigmoid: $\mathrm{F}(\mathrm{x})=\frac{1}{1+e^{-x}}$;
c) Bipolar Sigmoid Function: $\mathrm{F}(\mathrm{x})=\frac{1-e^{-x}}{1+e^{-x}}$;
d) Logistic symmetric: $\mathrm{F}(\mathrm{x})=\frac{1+e^{-x}}{2}$;
e) Hyperbolic tangent: $\mathrm{F}(\mathrm{x})=\tanh x=\frac{\sinh x}{\cosh x}=\frac{e^{x}-e^{-x}}{e^{x}+e^{-x}}$;
f) Corrected tangent: $\mathrm{F}(\mathrm{x})=\frac{1-e^{-2 x}}{1+e^{-2 x}}$;
g) Sinusoidal: $\mathrm{F}(\mathrm{x})=\sin (\mathrm{x})$;
h) Gaussian: $\mathrm{F}(\mathrm{x})=e^{-x^{2}}$;
i) Inverse Gaussian: $\mathrm{F}(\mathrm{x})=1-e^{-x^{2}}$;
j) $\operatorname{ReLu}: \mathrm{F}(\mathrm{x})=\max (0, \mathrm{x})$;
k) Softplus function $=\ln \left(1+e^{x}\right)$; 
The output of activation functions varies according to their type(Wang et al.., 2020). However, it is usually a result that oscillates, as displayed in Fig 3., between constant values, and that follows one of the most commonly used types of curves:
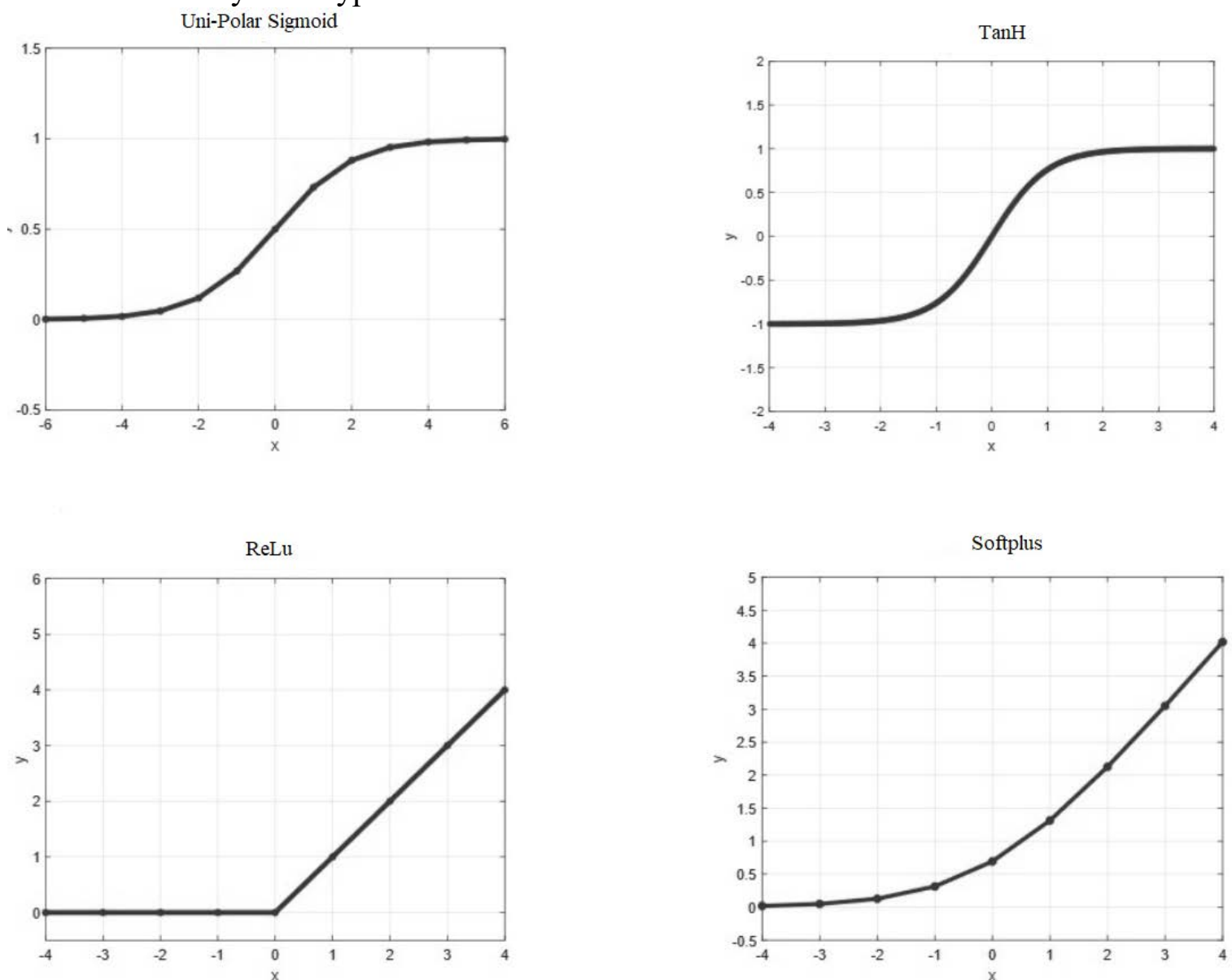

Figure 3. Output Graphs for Uni-Polar, TanH, ReLu and Softplus activation functions Source: adapted from The Influence of the Activation Function in a Convolution Neural Network Model of Facial Expression Recognition (Wang et al., 2020)

Forward propagation usually favors the ReLu function as a best practice, and the concept of transfer learning, as displayed in Fig. 4, unlocks convolutional neural networks' ability to make use of already learned knowledge, reducing the needed amount of new raw data to train models.
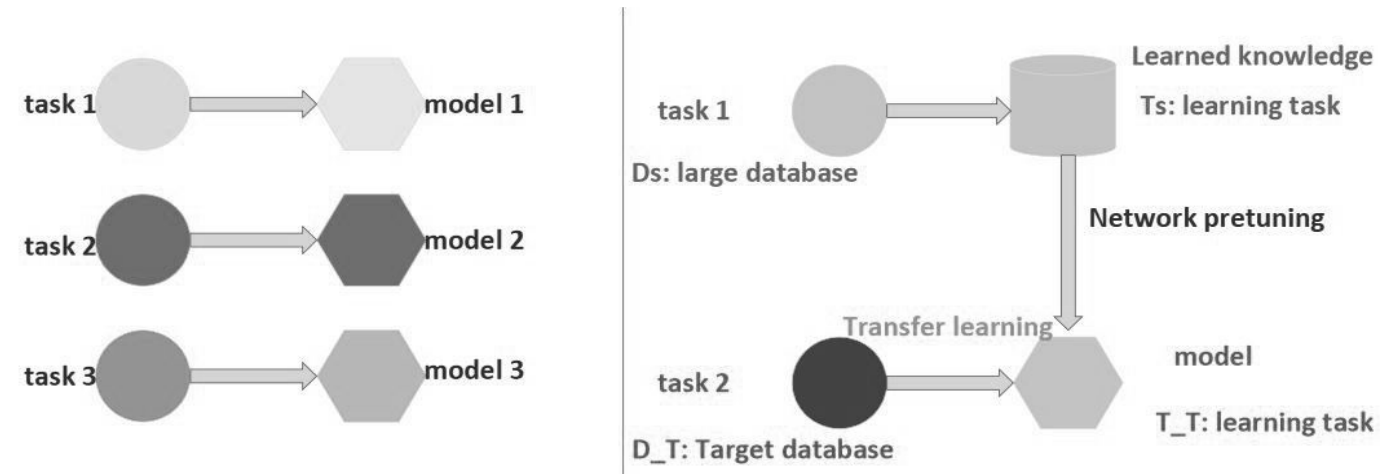

Figure 4. Traditional (left) and Transfer Learning (right)

Source: adapted from The Influence of the Activation Function in a Convolution Neural Network Model of Facial Expression Recognition (Wang et al., 2020) 


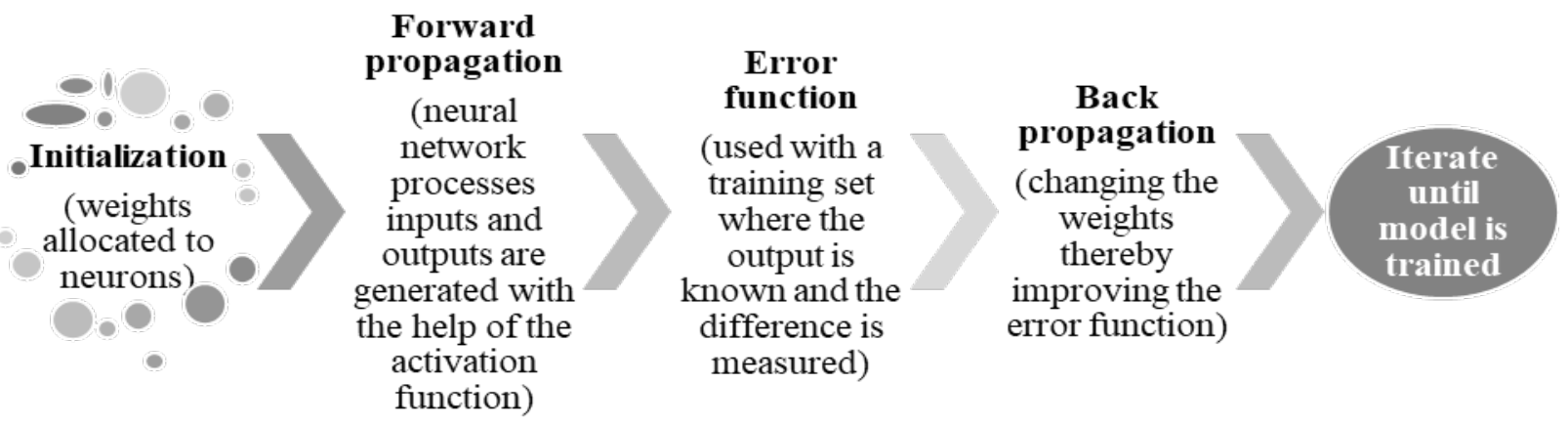

Figure 5. Process of training an artificial neural network

One of the most common methods of improving a neural network as displayed in Fig. 5 uses a method called backpropagation and updating weights by using a gradient descent force to minimize global loss function (Abdulkadir et al., 2013).

\subsection{Multi Agent Systems, basics and classification}

Components of multi-agent systems are strongly related to the type of action and interaction with their environment that may occur through sensors or digital data ingestion, digital outputs, or actuators in case of physical interaction. Decentralization, distribution, and interoperability provide an improved basis for autonomous systems capable of harnessing computing power, making it agile, more reliable, effective, and efficient.

Agent components consist of four main categories (Srinivasan \& Jain, 2020):

- Perception/data ingestion

- Reasoning/learning

- Action/result

- Communication/negotiation

\begin{tabular}{|c|c|c|c|c|c|c|c|c|c|c|}
\hline \multicolumn{5}{|c|}{ Perception } & \multirow{4}{*}{ 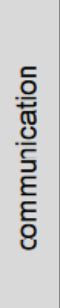 } & \multirow{2}{*}{\multicolumn{5}{|c|}{ Reasoning / Inference engine }} \\
\hline \multicolumn{5}{|c|}{ Reasoning / Inference engine } & & & & & & \\
\hline Reactive & $\begin{array}{l}\text { Belief } \\
\text { Model }\end{array}$ & Goals & History & utility & & Reactive & $\begin{array}{l}\text { Boliof } \\
\text { Model }\end{array}$ & Goals & History & utility \\
\hline \multicolumn{5}{|c|}{ Action } & & \multicolumn{5}{|c|}{ Action } \\
\hline
\end{tabular}

Figure 6. General Agent Concepts and Components

Source: adapted from An Introduction to Multi-Agent Systems (Srinivasan \& Jain, 2010)

According to the collective scope, the goals of an agent can be in various forms and numbers, single or multiple. Machine learning capabilities can then classify those goals into specific goals, adaptive goals such as reactive providing recommendations based on events and consequences, or pro-active 
based on data analysis and by using predictions. In neural networks, agents possess human-like behavior, which elevates their ability to incorporate neighbour knowledge, self-awareness, and negotiation with other agents. From a feature perspective, agents can be divided into following categories:

Table 1. Types of multi agent systems based on different feature classification

\begin{tabular}{|l|l|}
\hline Feature & Types \\
\hline Mobility & Fixed or mobile \\
\hline Output frequency & $\begin{array}{l}\text { Time dependent (fixed) } \\
\text { Event dependent (flexible) }\end{array}$ \\
\hline Topology & Static or dynamic \\
\hline Delay & With or without delay \\
\hline Leadership & Leader/Follower structure or independent \\
\hline Decision making & Linear or non-linear \\
\hline Heterogeneity & Heterogeneous or Homogenic \\
\hline
\end{tabular}

Source: adapted from Multi-Agent Systems: A survey. (2018)

As displayed in Table 1, the classification of multi agent systems cand be done in many ways considering the particular features that these may have (Dorri et al., 2018).

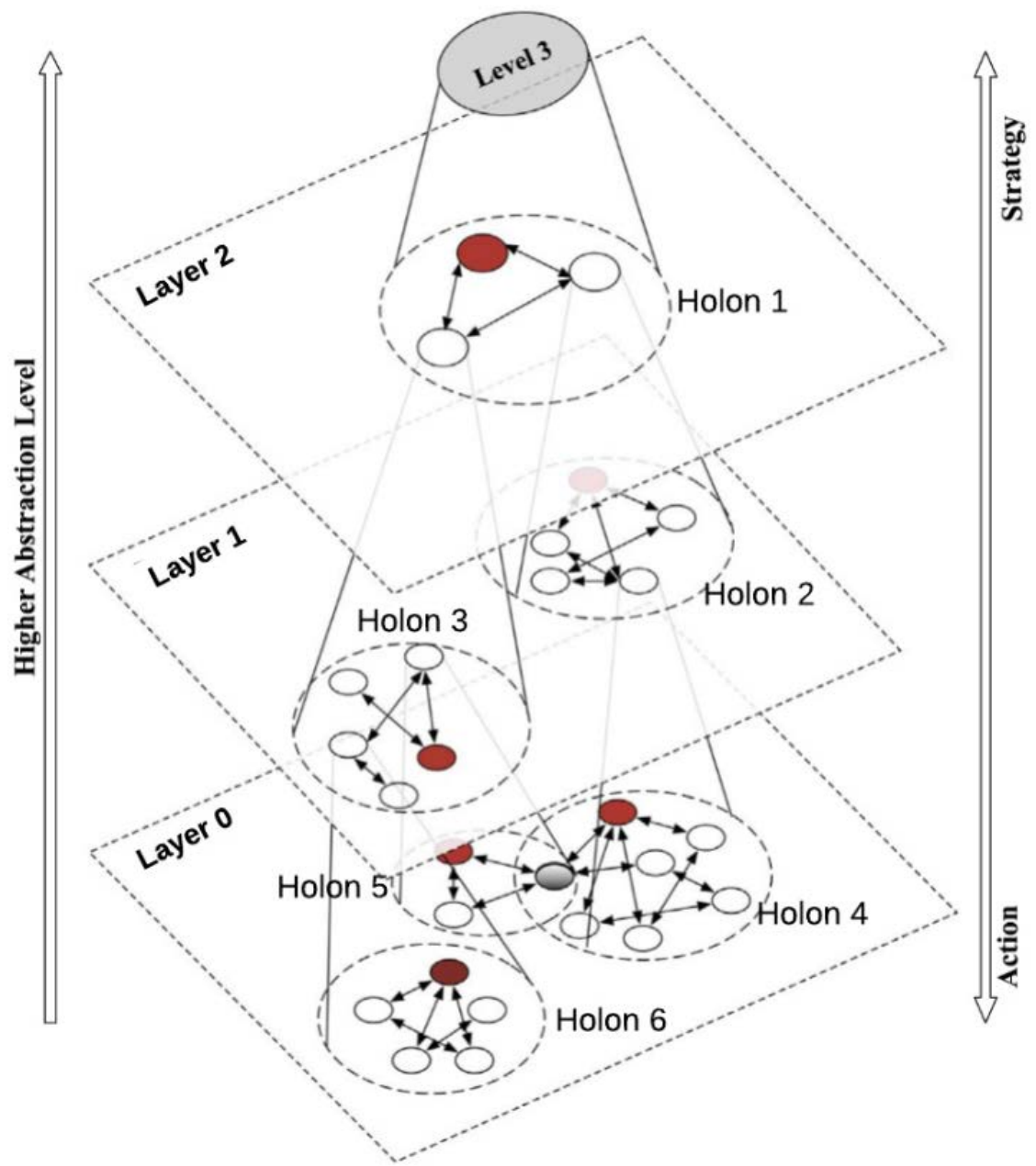

Figure 7. Holonic multi agent arhitecture

Source: adapted from Multi-Agent Systems: A survey. (Dorri et al., 2018) 
Based on organization methods, agents are a flat organization in homogeneous environments where all agents are similar in scope and type, hierarchical organizations where each agent has his parent and follows its bias. Most common architectures for multi agent systems are:

$>$ Flat

$>$ Hierarchical

$>$ Coalition

$>$ Congregation

$>$ Team

$>$ Matrix structures

In a scenario of using machine learning and multi-agent systems, a holonic structure represents the multi-layer framework of a neural network, as displayed in Fig. 7. Multi agents form groups of structures called holons, multi-agent systems aim for the different categories of inputs, analysis, and results. Agents can communicate in two dimensions, with other agents within the same holon or with other holons within the same layer. Therefore, an agent can interact with more holons within the same layer.

Learning of neural networks within a multi-agent system environment can be done in three ways: pro-active, re-active, or based on consequence. Communication within a Multi-Agent System is done through Knowledge Query and Manipulation Language (KQML) and consists of messages formed of three layers: communication, message, and content layer. Within the concept of Blackboards, agents perform different types of communication and negotiation and play various roles in the environment, such as a mediator, or facilitator, or leader who distributes tasks or smaller goals to reach a global goal.

\section{MANAGEMENT ASPECTS OF MULTI AGENT SYSTEMS}

Data-driven organizations are fully aware of data breaches and cybercrime. While a minute of downtime costs, on average, $\$ 8,851 /$ minute in the enterprise space and $\$ 282$ within the small and medium business segment, decision-makers are continuously engaged in essential topics such as governance, high-availability, security, and cost (Ponemon Institute, 2016). The adoption of multiagent systems can be a complicated task when dealing with human-centric, data-rich environments. Accessibility and availability of data influence the speed, accuracy, and complexity of a data science project. As highlighted in Fig. 8, multi-stakeholder collaboration is required within a broader range of aspects such as bias, ethics, regulatory compliance, and social aspects in a framework that includes technology, cost, and control.

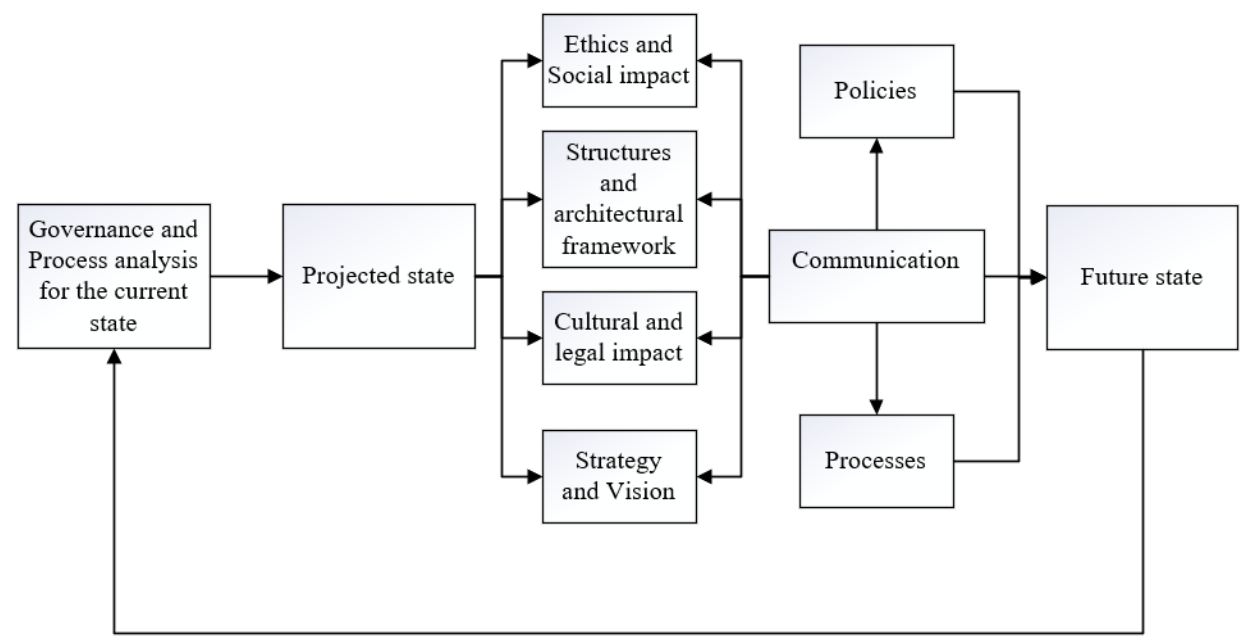

Figure 8. Governance process of adopting multi-agent systems 


\subsection{Agile Software Development Methodology of Adopting Multi-agent Systems}

By using Scrum and GAIA methodology, a popular method within the agile software programming space, teams can leverage a good efficiency and effectiveness throughout the whole software development process, and the development cycles or sprints (Sharma et al., 2012), as displayed in Fig. 8 maximum timeframe of 30 days. The main advantages of this method are (Gunga et al., 2013):

- Stakeholder satisfaction through the active involvement of stakeholders in the development process

- High adaptability to a fast-paced environment

- Reduced amount of documentation needs

- Reduced risk of errors

Disadvantages of this method can also be considered and are highly dependent on:

- People involvement and skillset

- Time constraints and documentation

- Every stakeholder change can over-utilize resources

- Pressure on developers for maintaining project timelines despite the constant change needs

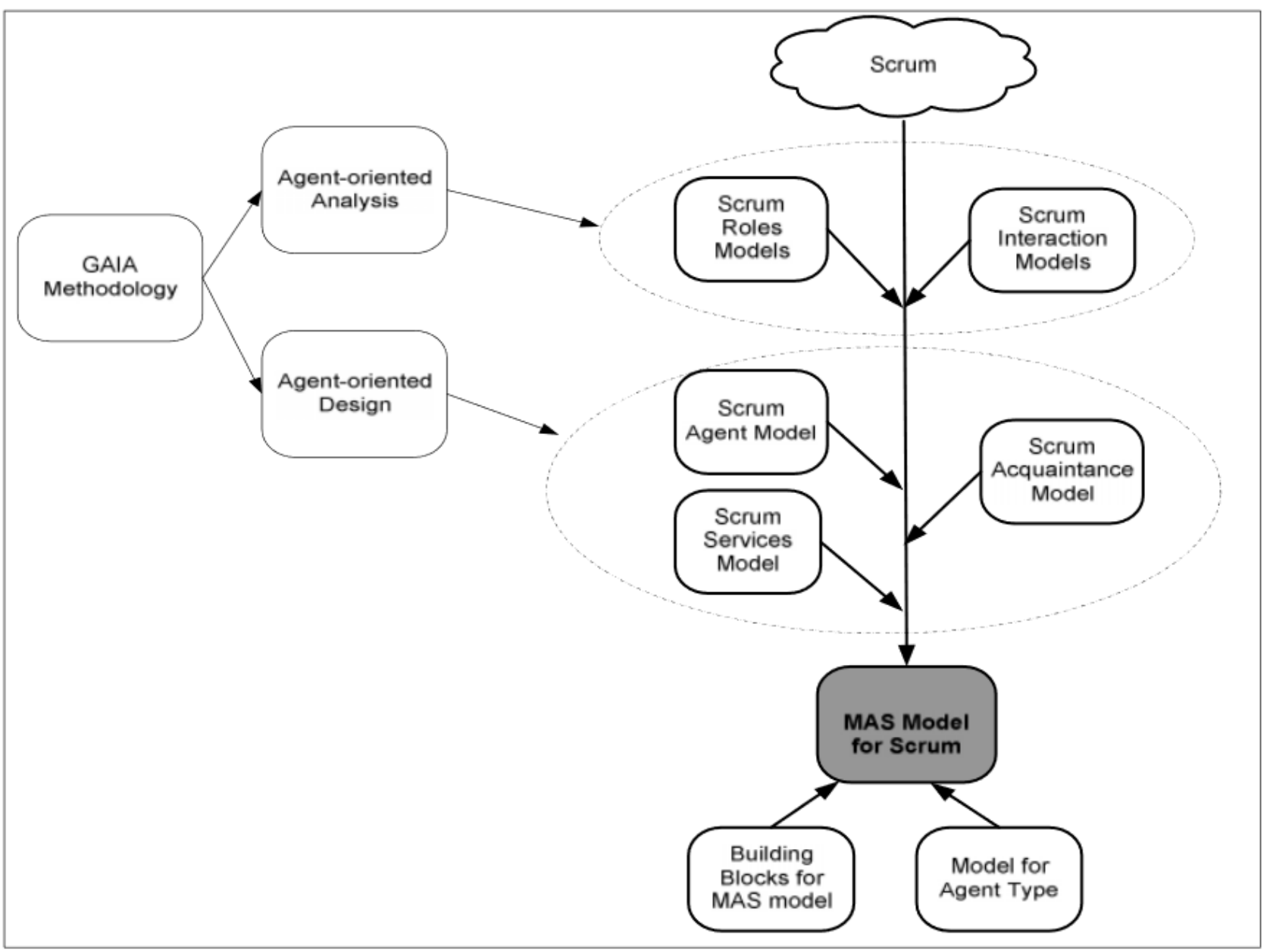

Figure 9. GAIA Methodology for Development of an Agile Process using Scrum Source: adapted from Design of a Multi-agent System Architecture for the Scrum Methodology (Gunga et al., 2013) 


\subsection{Architectural Framework}

Developed by the US Federal Government in 2006 and combining Zachman and TOGAF Frameworks, the Federal enterprise architecture framework, as displayed in Fig. 9, combines an adequate foundation combining the main pillars related to business, data, applications, and technology. Going forward, continuous development and continuous integration structure of an enterprise architecture emerge from having a clear focus on an evolutionary setup that integrates continuous, distributed change into core functions. Technology, applications, and data provide a clear foundation of the framework that facilitates the organization's digital transformation through the simultaneous elements focused on service delivery, functional integration, resource optimization, and authoritative reference. Different programs leverage the benefits of governance, principles, methods, tools, use, reporting, and audit and provide significant results through collaborative planning (The White House president Barack Obama, 2013).

Principles are:

- Future-ready

- Investment support

- Shared services

- Interoperability standards

- Data availability

- Security

- Technology inclusion

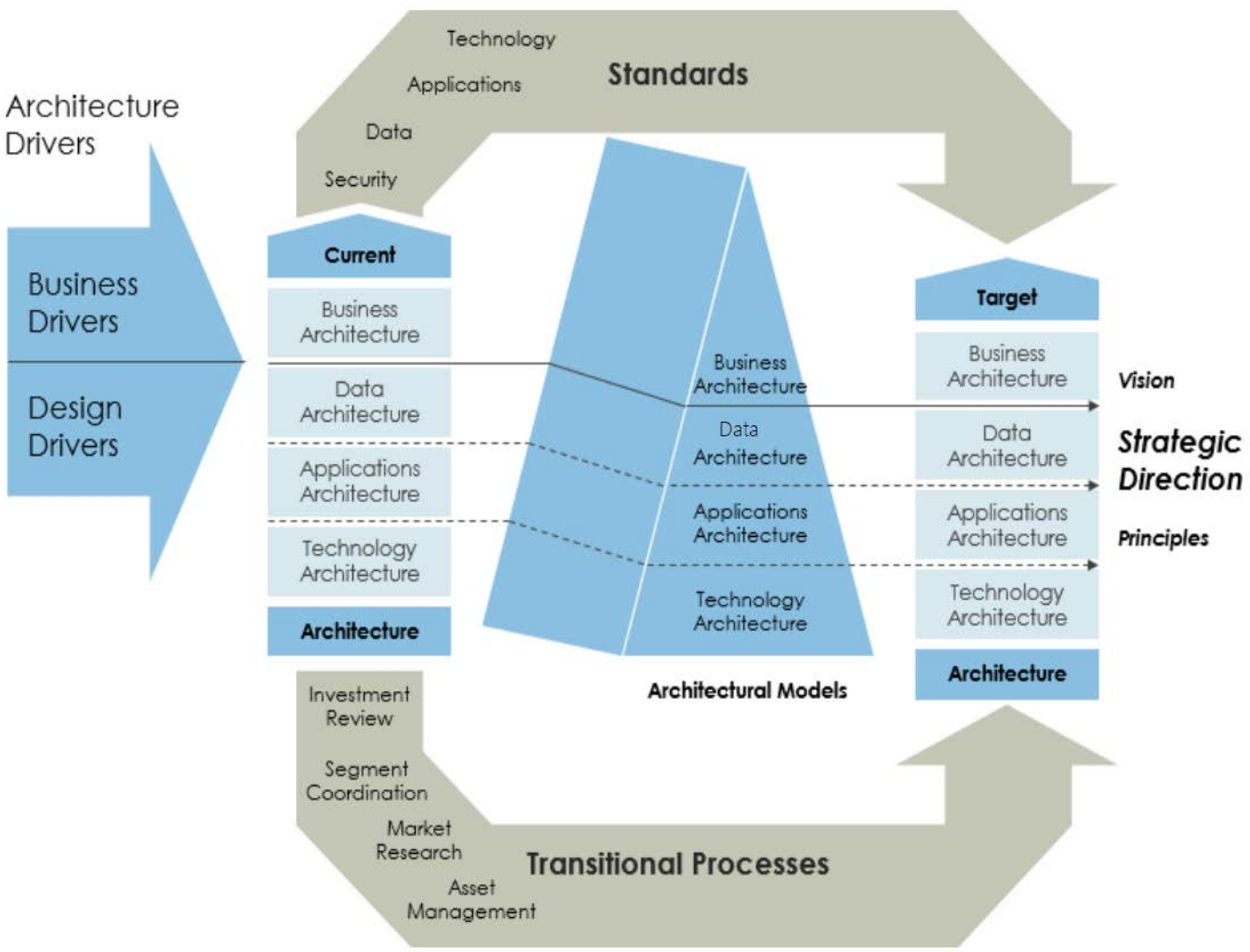

Figure 10. Federal Enterprise Architectural Framework

Source: adapted from Visual Paradigm (The White House president Barack Obama, 2013) 


\subsection{Technology, Applications, Data: The Big Data, Cloud-First, Shared Services Implementation Approach}

Distributed artificial intelligence requires a digital strategy for every aspect of the underlying framework components of technology, applications, and data. A coherent framework uses intangible resources paired with a unified technology layer across all segments, from data ingestion, edge pre-processing, to analytics and machine learning insights. Distributed computing, distributed storage in conjunction with heterogeneous computing, leads to the imperative of using a cloud platform to eliminate management bottlenecks, performance degradation and facilitate dynamic resource sharing through data center consolidation and shared services. Each application involving intelligent multi-agent systems utilizes the distributed computing and storage layers, as displayed in Fig. 10. Field programmable gate arrays (FPGA) support the computational capacity with an increase in capacity for kernel function acceleration by using hardware description language (HDL) the same way as application-specific integrated circuits (ASIC). Storage layers split into multiple storage classes according to the different throughput, and latency needs serving the different application types using data lakes, structured data, and in-memory storage. A combination of analytics and real-time processing demands Big data workloads like Apache Hadoop, including MapReduce and Apache Spark, to access multiple data sources simultaneously through a multimodel database system. Apache Spark also provides structured data models called resilient distributed data sets (RDD) with an in-memory approach. An intelligent file system requires the capability to move workloads through all storage layers, including random access memory (RAM), solid-state disks (SSD) or hard disks (HDD). Graphical processing units (GPU) enhance the distributed computing framework's capacity by exponentially outperforming central processing units (CPU) for convolution neural network training and real-time processing. Containerized applications for Platform-as-a-Service models increase the hardware and software resources utilization through solutions such as Docker, thereby reducing the footprint and cost. Open Computing Language (OpenCL) enables through functions named kernels a flexible execution between CPU and FPGA of computational workloads in a heterogeneous platform. It envisions a programmable application interface (API) that favours applications running on hosts to ignite kernels on heterogeneous multi-layer technology devices such as IoT, Edge, or Fog computing and manage their memory.

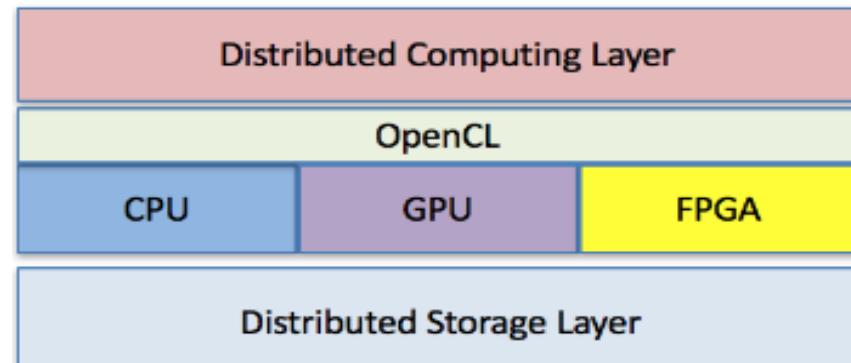

\section{Figure 11. Distributed Big Data Infrastructure of a Data Management and Analytics Cloud Platform}

Source: adapted from Liu et al., 2017

\subsection{Ethics and Social Impact}

Artificial intelligence within a multi-agent system should always be human-centric and comply with internationally recognized human rights and increase humans' wellbeing. It has to have the ability to add value to the human factor and enable people to choose their identity factors willingly. It has to be effective and efficient in reaching its purpose and transparent with all the decision-making, 
prediction, or reaction. Accountability aspects need to be regulated so that each decision can be traced and clear for all involved people. Also, humans need to be aware of the misuse of artificial intelligence. Clear guidelines should be in place for the purposeful usage of a mixed human and artificial intelligence environment.

In contrast with transparency, artificial intelligence has also to be secure. Therefore an "ethical black-box" capability needs to be in place for review and access all decision-making conditions that occur in a post-consequence manner. EU regulation 2016/679, systems have to ask for the consent of data usage, including timestamps (European Parliamentary Research Service, 2020). Safety and trust are seen as a fundamental principle for artificial intelligence development and recommends a framework for integrity and communication. Social harm and social justice have to include a nonbiased approach for the human factor in an inclusive environment and eliminate discrimination. Ethical and societal standards documented under ISO/IEC AWI TR 24368 provide clear guidance (ISO, 2020).

\subsection{Cultural and Legal Impact}

Processes and values have a high impact on culture (Mcadam \& Mitchell, 1998). Therefore, society's emotional influence has broad implications among different cultures, and the ways of interacting with artificial intelligence algorithms are influencing people and society itself. The importance of dialogue occurs while creating a culture of cooperation, openness, and trust of all involved layers of society, starting with the designers, engineers, and users of multi-agent artificial intelligence systems. Through responsible research and innovation, the EU facilitates a set of tools to assist every cultural and legal aspect of any stage a data science project involving multi-agent systems, and artificial intelligence occurs. From a legal perspective, artificial intelligence, including multi-agent systems, should not be categorized as a 'person'. However, it should comply with all international and domestic laws, and human control and regulation need to be in place at all times. Governments aim to cooperate with all actors ranging from society, industry, and regulators accordingly. The EU legal framework includes general product safety Directive 2001/95/EC, Directive 2000/43/EC, Directive 2000/78/EC, Directive 2004/113/EC, Directive 2006/54/EC, and unfair commercial practices and consumer rights directives Directive 2005/29/EC and Directive 2011/83/EC. Socio-economic impact includes business-education support, training, digital skills and competencies in science, engineering, mathematics, statistics and programming, and the need for modernization in the educational segment. A legal framework is provided by the European Commission and published as a White Paper on artificial intelligence on Feb 19, 2020.

\subsection{Strategy and Vision}

Every artificial intelligence-based project needs to be routed in a set of principles to ensure humancentricity and trust in artificial intelligence. The key highlights of this element envision critical elements according to the European Commission guidelines:

- Human-centric

- Technical accuracy and safety

- Privacy and Data Governance

- Transparency

- Diversity, non-bias, and fairness

- Sustainability and well-being

- Accountability (European Commission, 2020).

While the strategy can easily deflect measurable goals and objectives from the organization's overall vision, in an era where artificial intelligence disrupts all industries and segments, the need for aligning corporate vision with the individual, measurable goals are imperative. The implementation of artificial intelligence through multi-agent systems requires a team of diverse individuals ranging from research and development to commercial, financial, and production 
focused engineers (El Namaki, 2019). Customer-centric key performance indicators reveal the need for artificial intelligence to have a human-centric approach. Whereby the development of neural networks requires a constant change environment, choosing the right technology partners is a crucial aspect of an ever-increasing innovation demand where continuous learning and improvement occur.

\section{CONCLUSIONS}

The fifth industrial revolution stands out as an artificial intelligence-driven phenomenon. The enterprise industry standard for artificial intelligence and the multi-agent systems scales from corporate processes to small businesses and governments' adoption. Using multi-agent systems in artificial intelligence has several benefits:

- Better speed and operations efficiency and effectiveness through parallel computation and asynchronous operations

- Failure tolerance increasing high-availability

- Reduced cost in a decentralized architecture and heterogeneous computation (Korolov, 2019).

Across segments, the most common use cases are present in manufacturing and automotive, financial services, consumer goods, and retail and pharma and healthcare where artificial intelligence occurs:

- up to $60 \%$ quality and control

- up to $47 \%$ inventory management

- up to $36 \%$ personalization of products

- up to $52 \%$ fraud security and detection

- up to $48 \%$ customer care

- $\quad$ up to $29 \%$ pricing (MIT, 2020).

Challenges within the adoption of artificial intelligence appear from integrating with unstructured data, interacting with open-source platforms, eliminating bias from models, fulfilling regulatory compliance frameworks, and lacking data. Since $66 \%$ of organizational decision-makers are willingly ready to share internal data with third parties, data sharing unlocks organizations' potential for joint artificial intelligence development, thus increasing effectiveness, efficiency, and sustainability within the common processes (MIT, 2020). Distributed artificial intelligence emerges as a response o answer to future product and service innovations and is required to be:

* Human-centric

* Data-driven

* Agile

* Collaborative

The adoption of multi-agent systems that initiate a good foundation for the development of neural networks at scale envisions a strong, agile process strategy with a sprint approach, an enterprise framework with a joint infrastructure, application and data vision through ISO standards such as:

ISO/IEC WD TS 4213 Assessment of machine learning classification performance

ISO/IEC WD 5059 Quality Model for AI-based systems

ISO/IEC WD 5339 Guidelines for AI applications

ISO/IEC WD 5392 Reference architecture of knowledge engineering

ISO/IEC AWI TR 5469 Functional safety and AI systems

ISO/IEC TR 20547-1:2020 Big data reference architecture — Part 1: Framework and application process

ISO/IEC 20547-3:2020 Big data reference architecture - Part 3: Reference architecture

ISO/IEC TR 20547-5:2018 Big data reference architecture — Part 5: Standards roadmap

ISO/IEC CD 23053 Framework for Artificial Intelligence (AI) Systems Using Machine Learning (ML) 
ISO/IEC AWI TR 24027 Artificial Intelligence (AI) - Bias in AI systems and AI aided decision making

ISO/IEC TR 24028:2020 Artificial intelligence - Overview of trustworthiness in artificial intelligence

ISO/IEC CD TR 24368 Artificial intelligence - Overview of ethical and societal concerns

ISO/IEC AWI 24668 Artificial intelligence —Process management framework for Big data analytics

ISO/IEC CD 38507 Information technology - Governance of IT - Governance implications of the use of artificial intelligence by organizations

ISO/IEC WD 42001 Information Technology - Artificial intelligence - Management system

Standardization and collaboration are key aspects of future research as they provide interoperability and cost savings that increase resource utilization, generate a more sustainable environment, and accelerate collective evolution.

\section{REFERENCES}

Abdulkadir, T.S., Salami A.W., \& Kareem, A.G., (2012). Artificial Neural Network Modelling of Rainfall in Ilorin, Kwara State, Nigeria. Journal of Research Information in Civil Engineering, 9(1), Retrieved August $\quad 22, \quad$ 2020, from https://www.academia.edu/6735937/Artificial_Neural_Network_Modeling_of Rainfall_in_Ilor in Kwara State Nigeria

Dorri, A., Kanhere, S. S., \& Jurdak, R. (2018). Multi-Agent Systems: A survey. IEEE Access. Retrieved August 22, 2020, from https://www.researchgate.net/publication/324847369 MultiAgent Systems A survey

El Namaki, M. S. S. (2019). Will Artificial Intelligence Change Strategic Top Management Competencies? SCHOLEDGE International Journal of Management \& Development, 6 (4), 3447. Doi: http://dx.doi.org/10.19085/journal.sijmd060401. Retrieved August 20, 2020, from https://www.researchgate.net/publication/334400620_Will_Artificial_Intelligence_Change_Str ategic_Top_Management_Competencies

European Commission (n.d.). (2020). White Paper on Artificial Intelligence. A European approach to excellence and trust Retrieved August 29, 2020 from https://ec.europa.eu/info/sites/info/files/commission-white-paper-artificial-intelligencefeb2020 en.pdf

European Parliamentary Research Service. (2020). The Ethics of Artificial Intelligence: Issues and initiatives. $\quad$ Retrieved August $\quad 29,2020$ from https://www.europarl.europa.eu/RegData/etudes/STUD/2020/634452/EPRS_STU(2020)63445 2 EN.pdf

Farhadi, F. (2017). Learning activation functions in deep neural networks. Mémoire présenté en vue de l'obtention du diplôme de maîtrise ès sciences appliquées (Génie industriel), Université de Montréal. $\quad$ Retrieved August 16, 2020, from https://publications.polymtl.ca/2945/1/2017 FarnoushFarhadi.pdf

The White House president Barack Obama, (n.d.) (2013), Federal Enterprise Architecture Framework Version 2, Retrieved August 28, 2020 from https://obamawhitehouse.archives.gov/sites/default/files/omb/assets/egov_docs/fea_v2.pdf

Gunga, V., Kishnah, S., \& Pudaruth, S. (2013) Design of a multi-agent system architecture for the scrum methodology. International Journal of Software Engineering \& Applications (IJSEA), 4
$(4)$,
Doi:
$10.5121 /$ ijsea.2013.4401
Retrieved
August
28
from http://airccse.org/journal/ijsea/papers/4413ijsea01.pdf 
Korolov, M. (2019), Anatomy of an enterprise-scale AI strategy in CIO.com. Retrieved August 29, 2020 from https://www.cio.com/article/3408779/anatomy-of-an-enterprise-scale-aistrategy.html

Liu, S., Tang, J., Wang, C., Wang, Q. \& Gaudiot J.L. (2017). Implementing a Cloud Platform for Autonomous Driving, Retrieved August 28 from https://www.researchgate.net/publication/315882361_Implementing_a_Cloud_Platform_for_A utonomous Driving

MIT Technology Review Insights. (n.d.). The global AI agenda: Promise, reality, and a future of data sharing, In technologyreview.com. Retrieved March 26, 2020, from https://www.technologyreview.com/2020/03/26/950287/the-global-ai-agenda-promise-realityand-a-future-of-data-sharing/

Mcadam, R., Mitchell, N. (1998). Development of a business process re-engineering model applicable to the public sector. Total Quality Management, 9 (4-5), 160-163.

Nurfikri, F. (2020). An Illustrated Guide to Artificial Neural Networks, Towards Data Science, Retrieved on August 16, 2020, from https://towardsdatascience.com/an-illustrated-guide-toartificial-neural-networks-f149a549ba74

Oustimov, A. \& Vincent, V. (2014). Artificial neural networks in the cancer genomics frontier, Translational Cancer Research, 3 (3), 191-201. Doi: 10.3978/j.issn.2218-676X.2014.05.01. Retrieved on August 16, 2020, from http://tcr.amegroups.com/article/view/2647/html

Ponemon Institute (n.d.). (2016). Data Center Performance Benchmark Series. Cost of Data Center Outages, Retrieved August 26, 2020 from https://planetaklimata.com.ua/instr/Liebert_Hiross/Cost_of_Data_Center_Outages_2016_Eng.p df

Sharma S., Sakar D., \& Gupta, D. (2012). Agile Processes and Methodologies: A Conceptual Study. International Journal on Computer Science and Engineering (IJCSE), 4 (5), 892-897. Retrieved on August 20, 2020, from https://www.researchgate.net/publication/267706023 Agile Processes and Methodologies A Conceptual Study

Shiruru, K. (2015). Neural Network Approach for processing Substation Alarms. International Journals of Power Electronics Controllers and Converters, 1 (1), 21-28. Retrieved on August 16, 2020, from https://www.researchgate.net/publication/319903709

Srinivasan, D., Jain, L.C. (2010). Chapter An Introduction to Multi-Agent Systems in Innovations in MASs and Applications - 1, 1-27 Berlin: Springer-Verlag Berlin Heidelberg. https://www.researchgate.net/publication/226165258_An_Introduction to_MultiAgent Systems

ISO, (n.d.). Standards by ISO/IEC JTC 1/SC 42 Artificial intelligence. Retrieved May 3, 2020 from https://www.iso.org/committee/6794475/x/catalogue/

Wang, Y., Li, Y., Song, Y., \& Rong, X. (2020). The Influence of the Activation Function in a Convolution Neural Network Model of Facial Expression Recognition, Applied Sciences, 10 (5), 1897; https://doi.org/10.3390/app10051897. Retrieved on August 16, 2020, from https://www.mdpi.com/2076-3417/10/5/1897 